\title{
Investigación e innovación como valor agregado para una educación superior de calidad.
}

Research and innovation as an added value for quality higher education.

Maribel del Rocío Paredes Cabezas. ${ }^{1}$, Mary Elizabeth Cruz Lascano. ${ }^{2}$ \& Efraín Velasteguí López $^{3}$

Recibido: 05-12-2017 / Revisado: 17-02-2018 Aceptado: 13-03-2018/ Publicado: 01-04-2018

\begin{abstract}
.
DOI: https://doi.org/10.33262/cienciadigital.v2i2.84

Higher education is obliged to include transversal axes within its educational model in order to guarantee quality, thus research, development and innovation are fundamental axes of a quality educational system for the integral development of the country. Innovation understood as the process of generating ideas with a useful application in social development in which higher education can generate added value.
\end{abstract}

The generation of added value is possible when the educational experience is aimed at enhancing competencies, to say developing knowledge, abilities and skills of students, through the efficient linkage between education, research, creativity and innovation.

Currently, universities have been pressured by the demands from the labor market, and industrial and business environments increasingly demanding and changing, then must work on a quality educational offer that links research, innovation with development and consequent generation of strategic economic value, which at the time gave rise to the paradigm education as a fundamental element for the creation of wealth, this paradigm understood and applied in developed countries, but not in developing countries like Ecuador. Where, although there has been a shift in

\footnotetext{
${ }^{1}$ Universidad Técnica de Ambato, Facultad Contabilidad y Auditoría, Ecuador, maribeldparedes@uta.edu.ec ${ }^{2}$ Universidad Técnica de Ambato, Facultad Contabilidad y Auditoría, Ecuador, me.cruz@uta.edu.ec ${ }^{3}$ Universidad Técnica de Cotopaxi, Facultad SIYA, Cotopaxi, Ecuador, luis.velastegui7838@utc.edu.ec
} 
education, they have not managed to generate the much-desired economic development so far.

Although it is true, high educational level continues to be an essential requirement for access to the labor market, this does not guarantee either employment or social mobility, to say that not is enough to achieve third and fourth level degrees, nevertheless these must necessarily be backed by quality standards.

Universities have faced a number of problems, including the limited allocation of resources, which restricts research and development, that is the reason why educational patterns of slow adaptation to the changing conditions of the social environment have been formed, on the other hand within the educational system have coexisted and in some cases have opposed conservative and innovative trends, making it difficult to break traditional paradigms in the formation of students. Coating many times the generation of new ideas or prototypes that far from encouraging creativity are perhaps limiting the ability to innovate and achieve the necessary change in education. We must seek mechanisms that place the university as the privileged space for innovation, the generation and dissemination of knowledge, for debate and the search for solutions, a space of mutual commitment to generate and share innovations aimed at satisfying the needs of social development.

So far it has not been possible to articulate the labor market and education because apparently these two sectors attend to different logics and dynamics, the educational system seeks to respond to academic professional commitment from its own tradition, arising imbalances between training and professional performance, for which this document intends to propose a model that links research, and innovation in a system of educational quality, that combines the series of ideological and pedagogical options, so that it is possible to train professionals capable of facing the challenges of the growing international competition, where knowledge plays a crucial role in economic and social development, through the optimization of resources in the production process and the inclusion of innovation, information technology and telecommunications

Keywords: Innovation, Added Value, Research, Quality, Education.

\section{Resumen.}

La educación superior está obligada a incluir ejes transversales dentro de su modelo educativo a fin de garantizar calidad, así entonces la investigación, el desarrollo y la innovación, constituyen ejes fundamentales de un sistema educativo de calidad para el desarrollo integral del país. Innovación entendida como el proceso de generación 
de ideas con una aplicación útil en el desarrollo social en cuyo caso la educación superior puede generar valor agregado.

La generación de valor agregado es posible cuando la experiencia educacional este dirigida a potenciar las competencias, es decir desarrollar conocimientos, habilidades, destrezas y capacidades de los estudiantes, mediante la vinculación eficiente entre educación, investigación, la creatividad y la innovación.

Actualmente, las universidades se han visto presionadas por las exigencias del mercado laboral, y por entornos industriales y empresariales cada vez más exigentes y cambiantes, se debe entonces trabajar en una oferta educativa de calidad que vincule la investigación, la innovación con el desarrollo y consecuente generación de valor estratégico económico, lo que en su momento dio origen al paradigma la educación como elemento fundamental para la creación de riqueza, este paradigma entendido y aplicado en países desarrollados no así en países en vías de desarrollo como Ecuador. En donde si bien ha existido un giro en la educación no han conseguido hasta el momento generar ese desarrollo económico tan anhelado.

Si bien es cierto, el alto nivel educativo continúa siendo un requisito esencial de acceso al mercado laboral, este no garantiza ni el empleo, ni la movilidad social, es decir no basta con alcanzar títulos de tercer y cuarto nivel, sino que estos deben necesariamente estar respaldados por estándares de calidad.

Las universidades han enfrentado un sinnúmero de problemas, entre ellos la limitada asignación de recursos, lo que restringe la investigación y el desarrollo, razón por la cual se han conformado patrones educativos de lenta adaptación a las condiciones cambiantes del entorno social, por otra parte al interior del sistema educativo han coexistido y en algunos caso se han contrapuesto las tendencias conservadoras y las innovadoras dificultando la ruptura de paradigmas tradicionales en la formación de los estudiantes. Coartando muchas veces la generación de nuevas ideas o prototipos que lejos de alentar la creatividad quizá están limitando la capacidad de innovar y conseguir el cambio necesario en la educación. Debemos buscar mecanismos que sitúen a la universidad como el espacio privilegiado para la innovación, la generación y difusión del conocimiento, para el debate y la búsqueda de soluciones, un espacio de compromiso mutuo para generar y compartir innovaciones orientadas a satisfacer las necesidades de desarrollo social.

Hasta el momento no ha sido posible articular el mercado laboral y la educación porque al parecer estos dos sectores atienden a lógicas y dinámicas distintas, el sistema educativo, busca responder al compromiso profesional académico desde su 
propia tradición surgiendo desajustes entre la formación y el desempeño profesional, por lo que este documento pretende tiene plantear un modelo que vincule la investigación, y la innovación en un sistema de calidad educativa, que combine la serie de opciones ideológicas y pedagógicas, a fin de que sea posible formar profesionales capaces de enfrentar los retos de la creciente competencia internacional, en donde los conocimientos juegan un papel crucial en el desarrollo económico y social, a través de la optimización de los recursos en el proceso productivo y la inclusión de la innovación, la informática y las telecomunicaciones.

Palabras Claves: Innovación, Valor Agregado, Investigación, Calidad, Educación.

\section{Introducción.}

Actualmente las universidades se han visto presionadas por las exigencias del mercado laboral, por lo que el presente trabajo, busca vincular la investigación y la innovación como ejes fundamentales de un sistema educativo de calidad, en el cual la experiencia educacional este dirigida a potenciar las competencias; es decir, desarrollar los conocimientos, habilidades, destrezas y capacidades tanto de los docentes como de los estudiantes, a decir de Ramos (2008) "La calidad del sistema educativo va unida a la innovación" además enfrenta grandes desafíos a fin de que todos los ciudadanos accedan a una educación de calidad (Marchesi, tedesco y Coll, 2010). Este trabajo va mucho más allá pues considera necesario la vinculación eficiente entre educación, investigación, e innovación, esta última entendida además por la urgente necesidad del uso eficiente de multimedia educativa, solo entonces será posible generar valor agregado en la educación, es decir alcanzar altos estándares de calidad.

\section{Objetivos.}

- Enfatizar el rol de la investigación y la innovación como ejes transversales para la generación de valor agregado en la educación, a través de la inclusión de modelos pedagógicos alineados a la investigación de problemas reales, puesta en marcha de soluciones creativas y acordes al entorno, así como el uso de multimedia educativa que facilite la capacidad del docente a investigar e innovar, con el fin de formar individuos aptos para enfrentar los retos de la sociedad.

- Combinar las características de la investigación y la innovación, con el rol del docente y el alumno innovador e investigador, a fin de que se interrelacione la serie de opciones ideológicas, pedagógicas y tecnológicas en la comunidad de aprendizaje, a fin de superar con éxito las exigencias de la creciente competencia internacional.

- Plantear una matriz que vincule la investigación y la innovación, como pilares fundamentales de un sistema educativo de calidad en la educación superior, capaz de 
formar profesionales idóneos, aptos para enfrentar los retos cada vez más exigentes en el mercado laboral, con miras al tan anhelado desarrollo social y económico.

\section{Marco Teórico.}

Esta revisión teórica sobre la investigación e innovación como valor agregado para una educación superior de calidad, se enfoca en resaltar los factores básicos de calidad en la educación superior, así como una matriz que vincule la investigación con la innovación, a fin de que las universidades sean capaces de garantizar calidad en la oferta educativa a través de implementar mejoras sustanciales en el proceso de enseñanza - aprendizaje, pero sobre todo la vinculación eficiente de la investigación e innovación como factores esenciales en la generación de calidad, en este contexto resulta oportuno parafrasear lo expresado por Harvey y Williams (2010) quienes consideran que la falta de consenso sobre el impacto del factor calidad como pilar fundamental en el futuro y dirección de la educación superior, sin lugar a dudas hasta el momento solo ha disminuido el potencial transformador de la educación superior en la conquista de una mejor calidad de vida y el tan anhelado desarrollo sostenible.

La educación superior tendrá un verdadero impacto en el desarrollo económico y social de un país, cuando sus graduados en forma creativa, logren con éxito hacer una transferencia de conocimientos al sector productivo (Comunian, Gilmore and Jacobi, 2015), solo entonces se podrá hablar de valor agregado y calidad en la educación. La promoción de la investigación vinculada con la innovación educativa, constituyen ejes fundamentales para el desarrollo integral del país (Álvarez et al, 2008). El objetivo es fortalecer el desarrollo de un sistema educativo de calidad, para lo cual se requiere de la formación y actualización permanente de los docentes, así como un inquebrantable trabajo en equipo, se requiere además de la dotación de recursos educativos, el fomento de la lectura y uso de bibliotecas, junto a la autonomía pedagógica, organizativa y de gestión (LOE, 2006), para una verdadera transformación del sistema educativo con miras al futuro, lo cual sin lugar a dudas beneficiará el desarrollo de la sociedad.

En este contexto, la innovación juega un papel fundamental, la misma que deberá ser entendida como el proceso de generación de ideas con una aplicación útil en el desarrollo social, en cuyo caso la educación superior puede generar valor agregado, el mismo que es posible cuando la experiencia educacional este dirigida a potenciar las competencias, es decir desarrollar los conocimientos, habilidades, destrezas y capacidades de los estudiantes, mediante la vinculación eficiente entre educación, investigación, e innovación (Álvarez et al, 2008)

La tarea de las Instituciones de Educación Superior es por tanto fomentar el intercambio de conocimiento entre la academia y la economía local. Para lo cual debe incorporar la innovación en el quehacer educativo, a fin de preparar suficiente capital humano creativo, 
por su parte Kimmel (1992) citado por Adamczyk, Bullinger and Möslein (2012) considera que las universidades deben ser capaces de brindar a "los estudiantes la oportunidad de conectar conceptos cientificos con aplicaciones del mundo real", es decir, resolver problemas reales a partir no de simulaciones, sino de datos extraídos directamente del entorno, por otra parte Windlin et al., (2005) citados por Adamczyk, Bullinger and Möslein (2012) consideran que "fomentar la creatividad y estimular el desarrollo de actitudes fundamentales" en los estudiantes permite en los mismos conseguir mejores resultados.

Un sistema educativo de calidad que genere valor agregado, debe por tanto incluir contenidos que respondan adecuadamente a lo que los individuos necesitan para desarrollarse como personas e influenciar positivamente en la sociedad. Actualmente las universidades se han visto presionadas por las exigencias del mercado laboral, es necesario entonces trabajar en una oferta educativa de calidad que vincule la investigación y la innovación en la generación de valor estratégico económico, dando origen al paradigma: educación como elemento fundamental para la generación de riqueza.

Por tanto, una educación de calidad según la OCDE (1995) citado por Márquez (2011) es aquella que "asegura a todos los jóvenes la adquisición de los conocimientos, capacidades destrezas y actitudes necesarias para equipararles para la vida adulta"

Así también Mortimore (1991) citado por (Márquez, 2011) manifiesta que educación de calidad: "es la que promueve el progreso de sus estudiantes en una amplia gama de logros intelectuales, sociales, morales y emocionales, teniendo en cuenta su nivel socioeconómico, su medio familiar y su aprendizaje previo". Si bien se requiere de un cambio profundo en el sistema educativo resulta un proceso complejo y largo, por lo que demanda de un proyecto global integrado, asumido por todos los agentes educativos, y que estos se articulen en una misma dirección, coherente y con objetivos educativos compartidos, es por tanto un trabajo conjunto y riguroso, a fin de que se fortalezca un sistema educativo eficaz en el que se maximice la capacidad de discentes y docentes para alcanzar resultados efectivos que trascienda en beneficio de la sociedad. Sin lugar a dudas, falta la toma de conciencia seria sobre lo que significa cambio educativo, ¿cómo se gestiona? y ¿cuál es el papel de los diferentes agentes y su vinculación?

Si bien es cierto, el alto nivel educativo continúa siendo un requisito esencial de acceso al mercado laboral, este no garantiza ni el empleo, ni la movilidad social (Gómez y Celis 2012), es decir, no basta con alcanzar títulos de tercer y cuarto nivel, sino que estos estén respaldados por estándares de calidad. En donde la innovación y la puesta en práctica del conocimiento creativo sean la diferencia. Así, parafraseando a Comunian et al. (2015) son precisamente las Instituciones de Educación Superior conocidas como los "terceros espacios" los llamados a generar el ambiente propicio para la transferencia de conocimiento creativo, lo cual no resulta una tarea fácil, pues se debe al mismo tiempo fomentar el 
conocimiento creativo y a la vez encajar en el entorno, a fin de dar solución a problemas sociales y de la economía en general.

Una educación de calidad constituye una herramienta para el cambio social, siempre que esté vinculada a la investigación y a la innovación, estos factores fundamentales en la educación a nuestro juicio se convierten en pilares que pueden sumarle valor agregado al sistema educativo y por tanto permiten el logro de objetivos. La educación superior deberá estar encaminada a proporcionar habilidades para: "aprender a conocer, aprender a hacer, aprender a ser y aprender a vivir juntos" (Scott, 2015 al referirse a los cuatro pilares de la educación mencionados en el Informe Delors, 1996), es decir que la educación debe ser capaz de facilitar a los individuos herramientas para el desarrollo de capacidades y habilidades que le permitan la comprensión de su entorno y la realidad que lo rodea, para contribuir a la transformación social y la posibilidad de una mejor calidad de vida. Interpretando a Blanco (2012), no podremos alcanzar educación con altos estándares de calidad, si no tenemos docentes de calidad, apreciación que concuerda con la expresada por Podder (2017) quien considera que "sin maestros de calidad, la educación de calidad siempre permanecerá muy lejos".

Las universidades han enfrentado un sinnúmero de problemas, entre ellos la limitada asignación de recursos, lo que restringe la investigación, el desarrollo y la capacitación permanente y oportuna de los docentes, razón por la cual se han conformado patrones educativos de lenta adaptación a las condiciones cambiantes del entorno social, por otra parte al interior del sistema educativo están presentes tendencias conservadoras e innovadoras que en ocasiones se han contrapuesto, dificultando la adopción de nuevos paradigmas en la educación (Levy, 2000). Debemos buscar mecanismos que sitúen a la universidad como el espacio privilegiado para la innovación, la generación y difusión del conocimiento, así como para el debate y la búsqueda de soluciones, un espacio de compromiso mutuo para generar y compartir innovaciones orientadas a satisfacer las necesidades de desarrollo social y económico.

El presente artículo de reflexión teórica, pretende poner énfasis en la innovación y la investigación, como pilares fundamentales de un sistema de calidad que genere valor agregado en los individuos, partícipes del sistema educativo. La calidad en la educación debe por tanto ser entendida como el derecho fundamental, que a más de ser eficaz y eficiente, relevante, pertinente y equitativa debe ante todo respetar los derechos de todas las personas, Blanco et al (2007). La investigación según Olivo y Montaño (2014): “debe ser entendida como el proceso por medio del cual se incrementa la visión analítica y profunda de la realidad, teniendo como resultado una aportación al bienestar social y (...) desarrollo intelectual" 
La innovación por su parte no debe ir en contradicción con los objetivos de la educación y el ajuste en el entorno de un sistema educativo de calidad; por tanto, la innovación se constituye en uno de los ejes primordiales para conseguir un desarrollo sostenible, en donde los actores de la innovación educativa estén relacionados entre sí.

Es prioritario que las políticas educativas estén dirigidas a potenciar la formación de los docentes a fin de que estos sean capaces de implementar cambios e innovaciones necesarias en su entorno, como respuesta a las nuevas exigencias del mercado laboral. Hasta el momento no ha sido posible articular el mercado laboral y la educación porque al parecer estos dos sectores atienden a lógicas y dinámicas distintas. El sistema educativo por su parte busca responder al compromiso profesional académico desde su propia tradición surgiendo desajustes entre la formación y el desempeño profesional. Surge entonces una "transferencia de responsabilidades" desde el mercado laboral hacia el sistema educativo (Martuscelli y Martínez, 2002 citado por Hernández y Rodríguez, 2015)

A decir de Hernández (2009) "es necesario fortalecer el desempeño de los docentes investigadores mediante la generación de espacios para la reflexión y la formación investigativa, en aras de una educación de calidad". En este contexto se debe proporcionar a los docentes de una formación científico-técnica, a fin de dotarlos de estrategias metodológicas que le permitan llevar a cabo innovaciones, propiciar el trabajo en equipo. Por otra parte, se requiere de un amplio apoyo social y político, mediante el cual sea posible conformar un sistema educativo de calidad, cuyo objetivo principal según Hopkins, 2001 citado por Harris et. al (2006) es conseguir que el sistema educativo es decir que las escuelas, colegios y universidades se convierta en verdaderas comunidades de aprendizaje donde todos aprendan día tras día, tanto los alumnos como los profesores y la sociedad en general.

Hernández (2009) además considera que la verdadera labor del docente investigador es formar profesionales competentes, quienes, con el apoyo de sus propias investigaciones, sean capaces de generar conocimiento válido que responda a múltiples problemas del entorno.

En este artículo se plantea un modelo que vincule la investigación y la innovación en un sistema educativo de calidad, el mismo que combina las características de la innovación y la investigación, con el rol del docente investigador, innovador y el alumno quien también debe demostrar su interés por la investigación e innovación. Todo esto con el fin de propiciar que la comunidad de aprendizaje interrelacione la serie de opciones ideológicas y pedagógicas generando valor agregado en la educación al ser capaces de enfrentar con éxito las exigencias del mercado laboral.

Es así que una vez colocados a la innovación y la investigación como pilares fundamentales, será posible cimentar un sistema educativo de calidad en la educación 


\section{Ciencia

superior capaz de formar profesionales idóneos, aptos para enfrentar los retos cada vez más exigentes en el mercado laboral, así como superar con éxito las exigencias de la creciente competencia internacional, en donde los conocimientos juegan un papel crucial en el desarrollo económico y social, que solo será posible con la optimización de los recursos en el proceso productivo y la inclusión de espacios para dar rienda suelta a la creatividad y la innovación; así como la inclusión de modelos pedagógicos "tecnológicamente mejorados" (Bell and Binns, 2013), gracias al empleo de multimedia educativa , generando así "una experiencia positiva de aprendizaje” Matusiak (2013) con resultados exitosos y de alto impacto en la sociedad y la economía local.

Grafico No1. Formas nuevos profesionales.

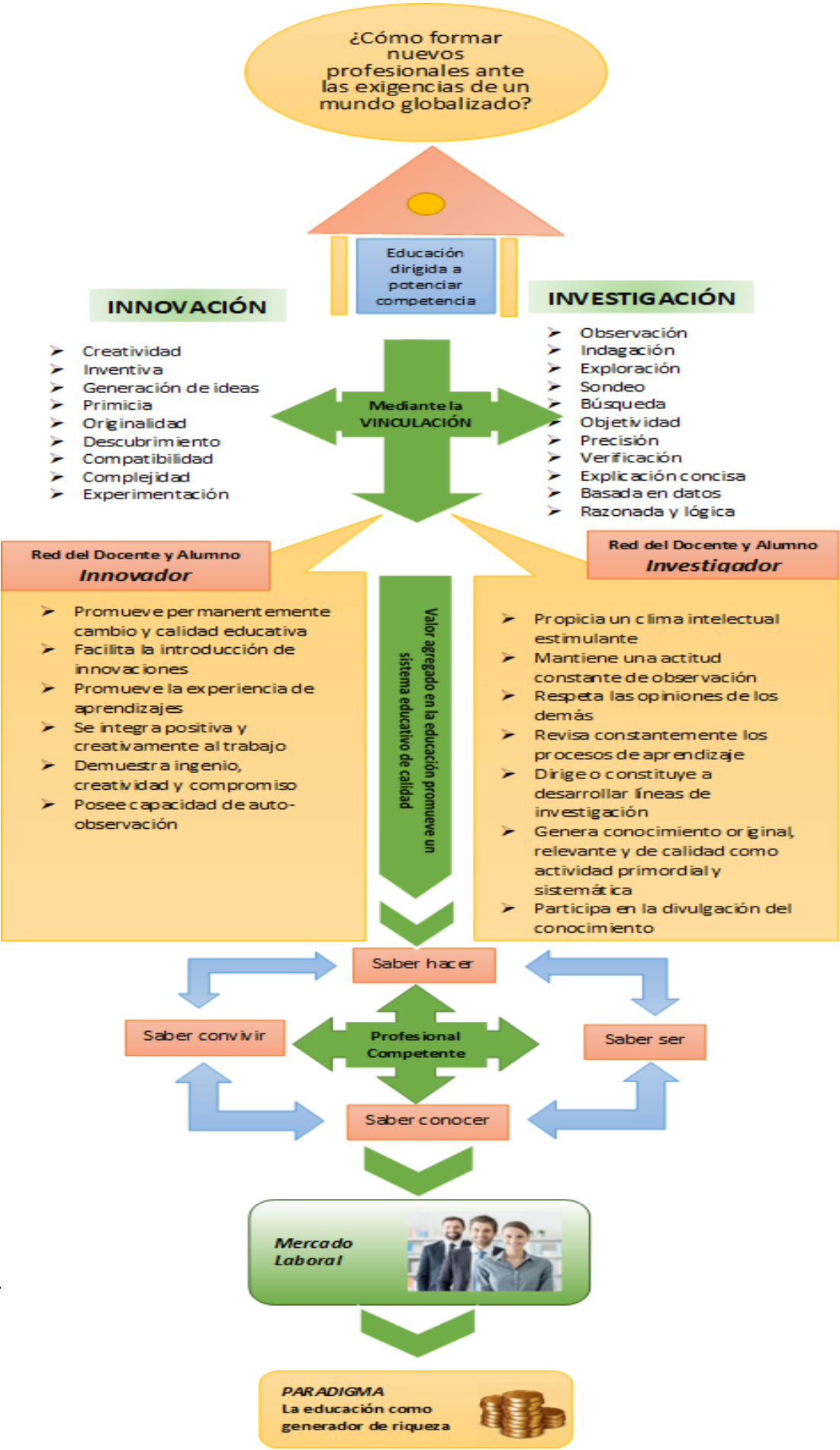


Elaborado por: Grupo de Investigación

En nuestro país, al parecer el gobierno está consciente de que el cambio empieza por la calidad en la educación superior y que no sólo basta con reconocer un problema teórico, sino asumir el hecho de que la calidad, es parte de un ineludible del compromiso profesional académico que consiste en poner a disposición de los actores un marco de decisión para generar valor agregado y excelencia en el proceso educativo.

El valor agregado en el sistema educativo se puede medir a través del desempeño competente de los nuevos profesionales en el campo laboral, para lo cual se requiere impulsar una educación de calidad, basada en la investigación, para lo cual será necesaria la participación de todos los actores involucrados, a fin de contar con docentes actualizados. Y la confluencia de distintos factores, tales como: contenidos adaptados al entorno y realidades locales, mejora sustancial de los procesos cognitivos, en donde la investigación y la innovación juega un papel fundamental; por su puesto se requiere del apoyo gubernamental, a fin de contar la infraestructura adecuada y la tecnología necesaria para propiciar procesos educativos de alta calidad.

El Plan Decenal de Educación 2016-2025 plantea tres objetivos primordiales de la educación, así objetivo de calidad, objetivo de cobertura y objetivo de gestión; precisamente el objetivo de calidad plantea la necesidad de "Educar integralmente para el desarrollo personal y la equidad social", basado en la "calidad educativa multidimensional, que garantice la excelencia (...) y contribuyan a alcanzar una sociedad democrática, armónica, intercultural, próspera, y con igualdad de oportunidades”.

Esto obliga a las universidades a diseñar modelos educativos que involucren la investigación y la innovación, así como el uso de multimedia educativa, además se debe contar con estrategias para la gestión eficaz de recursos. En este contexto puede también resultar interesante combinar políticas de incentivos a los profesores más competentes o más comprometidos con el quehacer educativo, a fin de motivarlos a dar lo mejor de sí en beneficio del modelo educativo adoptado.

Se debe por ejemplo realizar autoevaluaciones permanentes que permitan identificar fortalezas a fin de potenciarlas, por lo tanto, no todas las universidades podrán abordar con 
éxito todas las funciones de docencia e investigación, lo cual producirá inevitablemente una diferenciación en el nivel de calidad en la educación.

\section{Factores básicos de calidad en la educación superior.}

Tabla No1. Factores de calidad.

\begin{tabular}{ll}
\hline \multicolumn{1}{c}{ FACTORES A IMPULSAR } & FACTORES A ELIMINAR \\
\hline $\begin{array}{l}\text { Actitud y desempeño del } \\
\text { docente } \\
\text { Aptitud y actualización } \\
\text { permanente }\end{array}$ & Libertad de cátedra mal entendida \\
$\begin{array}{l}\text { Planificación curricular } \\
\text { actualizada y adaptada al } \\
\text { entorno }\end{array}$ & $\begin{array}{l}\text { Perfil del profesor no acorde a la } \\
\text { cátedra }\end{array}$ \\
$\begin{array}{l}\text { Impulsar la investigación y la } \\
\text { innovación }\end{array}$ & $\begin{array}{l}\text { Seguimiento y Evaluación sin } \\
\text { resultados }\end{array}$ \\
$\begin{array}{l}\text { Implementar mejoramiento } \\
\text { continuo }\end{array}$ & $\begin{array}{l}\text { Falta de pedagogía y/o } \\
\text { andragogía }\end{array}$ \\
$\begin{array}{l}\text { Fomentar el uso de multimedia } \\
\text { educativa }\end{array}$ & $\begin{array}{l}\text { Docentes desactualizados en su } \\
\text { cátedra o en el uso de tecnologías }\end{array}$ \\
\hline
\end{tabular}

Fuente: Elaboración propia a partir de Marqués (2011).

\section{Conclusiones.}

- Por tanto, la formación de profesionales en un mundo globalizado, con innovadoras formas de creación, almacenamiento y transmisión de información y conocimiento, plantea desafíos a las universidades, que deben actuar proactivamente ante la situación y no en forma reactiva como es usual, "en épocas de crisis solo la imaginación es más importante que el conocimiento”. (Albert Einstein)

- La educación superior, es un sector con alcances económicos y sociales incalculables en el desarrollo de una sociedad, esto implica forzosamente que las universidades estén obligadas a orientar sus actuaciones hacia las grandes tendencias sociales, cambiando sus esquemas tradicionales de organización de la enseñanza y de la investigación, adoptando nuevos métodos que aporten valor agregado al aprendizaje con la incorporación de las tecnologías de la información y de las comunicaciones, teniendo en cuenta las nuevas demandas y las nuevas necesidades en un mundo globalizado y competitivo. 
- En estos momentos de cambios profundos en los sistemas educativos, es imprescindible que las políticas educativas se dirijan a potenciar la formación del profesorado de cara a que sea él mismo quien realice los cambios e innovaciones necesarias en su entorno, y así dar respuesta a las nuevas exigencias sociales derivadas de su función educadora.

- Esto requiere por tanto dotar al profesorado de una formación científico-técnica, así como el empleo de herramientas tecnológicas y multimedia educativa, además de estrategias para llevar a cabo innovaciones. Se requiere también de un amplio apoyo social y político. Actualmente, los docentes necesitan saber enfrentarse a una nueva realidad, deben querer aprender, investigar, gustarles su trabajo, tener presente los fines de la educación, trabajar en equipo, ser reflexivos y flexibles en la aplicación de nuevas prácticas e innovaciones, no tener miedo a desarrollar experiencias para solucionar problemas; y, por supuesto, deben tener grandes dosis de optimismo y confianza en lo que hacen.

- Independientemente de las variaciones en el comportamiento de los mercados laborales, la economía en su conjunto ha sido incapaz de generar fuentes de empleo sostenido para la oferta de profesionales. Las posibilidades de ocupación de los egresados de la educación superior y media superior fluctúan en función de la dinámica de crecimiento económico, las universidades por su parte tienen que analizar su rol en la sociedad, el mismo que no sólo debe limitarse a proporcionar educación terminal, sino que debe revalorar su misión educativa de formar profesionales, su misión debe ir mucho más allá pues debe ser capaz de formar profesionales competentes con capacidad de pensar y aprender por sí mismos.

- Calidad en la educación superior universitaria implica reconocer que en lugar de un problema teórico, uno se enfrenta con el paradigma de distinguir que la calidad es parte de un ineludible compromiso profesional académico que implica poner a disposición de los actores un marco de decisión para lograr valor agregado y excelencia en el proceso educativo, El valor agregado en la experiencia educacional se puede medir a través del conocimiento, las habilidades y las destrezas de los estudiantes reflejadas en su ejercicio profesional; es decir, en el campo laboral sólo entonces la educación superior podrá estar segura de que ha formado profesionales competentes.

- En este contexto, las universidades tendrán que diseñar estrategias de búsqueda y de gestión de recursos para el desarrollo institucional, combinando políticas de incentivo para los departamentos y profesores más competentes. Dentro de la multiplicidad de funciones que las caracterizan, tendrán que examinar sus fortalezas y descubrir sus oportunidades ajustando sus actividades en función de ellas y de sus recursos. No todas las universidades podrán abordar con éxito todas las funciones lo que producirá inevitablemente una diferenciación en el nivel de calidad en la educación. 
- La educación universitaria no debe estar dirigida solo al dominio de contenidos, sino a la habilidad para reflexionar y aplicar el conocimiento; y al manejo de destrezas necesarias para conseguir objetivos personales tales como: contribuir a la transformación social y a satisfacer las necesidades de desarrollo económico, que propicie además una participación efectiva de los nuevos profesionales en la sociedad, finalmente que garantice la posibilidad de una mejor calidad de vida.

\section{Referencias bibliográficas.}

ADAMCZYK Sabrina, BULLINGER Andelika. and MÖSLEIN Kathrin (2012), Innovation Contests: A Review, Classification and Outlook. Creativity and Innovation Management, Vol. 21 Number 4: 335-360. doi:10.1111/caim.12003. [en línea]. Recuperado de: https://onlinelibrary.wiley.com/doi/epdf/10.1111/caim.12003

ÁLVAREZ, Carmen, CASTILLO Carmen, MÁRQUEZ Lorena, OLIVEROS Glenda, RODRÍGUEZ Milbia, VILLEGAS Catalina, VILLEGAS Carmen. (2008). Valor Agregado y Aprendizaje Organizacional. Innovaciones Tecnológicas y Educación. Pontificia Universidad Católica del Perú.

Lima - Perú. [en línea]. Recuperado de: http://comunidadtecnologica.blogspot.com/2008/02/valor-agregado-yaprendizaje.html.

BELL, R. L., MAENG, J. L. and BINNS, I. C. (2013). Learning in context: Technology integration in a teacher preparation program informed by situated learning theory. $\mathrm{J}$. Journal of Research in Science Teaching.50: 348-379. doi:10.1002/tea.21075. [en línea]. Recuperado de: https://onlinelibrary.wiley.com/doi/epdf/10.1002/tea.21075

BLANCO Rosa et al. (2007) "Educación de Calidad para todos: Un asunto de Derechos Humanos". UNESCO. Documento de discusión sobre políticas educativas en el marco de la II Reunión Intergubernamental del Proyecto Regional de Educación para América Latina y el Caribe (EPT/PRELAC) 29 y 30 de marzo de 2007; Buenos Aires, Argentina [en línea]. Recuperado de: http://unesdoc.unesco.org/images/0015/001502/150272s.pdf

BLANCO, Rosa (2012). Una atención y educación de calidad en la primera infancia puede hacer la diferencia. Revista Docencia Vol. 48. [en línea]. Recuperado de: http://www.revistadocencia.cl/new/wp-content/pdf/20121213213459.pdf 
COMUNIAN, R, GILMORE, A, and JACOBI, S (2015), Higher Education and the Creative Economy: Creative Graduates, Knowledge Transfer and Regional Impact Debates. Geography Compass, 9/7, 371-383. doi: 10.1111/gec3.12220. [en línea]. Recuperado de: https://onlinelibrary.wiley.com/doi/pdf/10.1111/gec3.12220

DELORS, Jacques (1996) La Educación encierra un Tesoro, informe a la UNESCO de la Comisión Internacional sobre la Educación para el siglo XX. Santillana Ediciones Unesco. ISBN 92-3-303274-4 [en línea]. Recuperado de: http://unesdoc.unesco.org/images/0010/001095/109590so.pdf

GÓMEZ CAMPO, Víctor Manuel y CELIS GIRALDO, Jorge Enrique (2012). Factores de Innovación Curricular y Académica en la Educación Superior. Universidad Nacional de Colombia. Revista Iberoamericana de Educación. ISSN: 1681-5653. [en línea]. Recuperado de: file://C:/Users/Docente01/Downloads/773Gomez\%20(2).PDF

HARRIS Alma, JAMES Sue, GUNRAJ Judith, CLARKE Paul and HARRIS Belinda (2006). Improving Schools in excepcionallity Challingin Circunstances. Brithish Library Catalogiun-in-Publication Data. London - England 157p. ISBN: 0-8264-7494-2.

HARVEY, Lee \& WILLIAMS, James. (2010) Fifteen Years of Quality in Higher Education (Part Two), Quality in Higher Education, ISSN 13538322, 14701081. Vol. 16, No. 2, July 2010, 16:2, 81-113, DOI: 10.1080/13538322.2010.485722 [en línea].

Recuperado de: http://www.qualityresearchinternational.com/Harvey\%20papers/Harvey\%20and\%2 0Williams\%2015\%20Years\%20Pt2.pdf

HERNÁNDEZ ARTEAGA, Isabel (2009). El docente investigador en la formación de profesionales. Revista Virtual Universidad Católica del Norte, (27), 1-21 Medellín Colombia. ISSN 0124-5821. mayo - agosto 2009. [en línea]. Recuperado de: http://www.redalyc.org/pdf/1942/194215432011.pdf

HERNÁNDEZ, Juan Manuel y RODRÍGUEZ, Javier (2015). La pertinencia de la educación desde la perspectiva de los estudiantes en una universidad pública mexicana. Cuadernos de Investigación Educativa, Vol. 6, № 1, 2015, Montevideo (Uruguay), 33-51. ISSN 1688-9304 Universidad ORT Uruguay. [en línea]. Recuperado de: file:///C:/Users/Docente01/Downloads/6-20-1-PB.pdf

LEVY VÁSQUEZ, Javier Carlos (2000). Propuesta de una nueva forma para enseñar y aprender psicología de la empresa. Universidad de Colima. Facultad de Pedagogía. Recuperado 
http://digeset.ucol.mx/tesis_posgrado/Pdf/Javier\%20Carlos\%20Levy\%20Vazquez.p df

MARCHESI Álvaro, TEDESCO Juan Carlos, COLL César. (2010) Calidad, equidad y reformas en la enseñanza. Metas Educativas 2021. La educación que queremos para la generación de los Bicentenarios. Madrid - España. 175p. ISBN: 978-84-7666195-6 Organización de Estados Iberoamericanos para la Educación, la Ciencia y la Cultura (OEI) Fundación Santillana [en línea]. Recuperado de: file:///C:/Users/Docente01/Downloads/CALIDAD\%20(1).pdf

MARQUÉS GRAELL, Pere. (2011). Calidad e Innovación Educativa en los Centros Departamento de Pedagogía Aplicada, Facultad de Educación, UAB. Barcelona España. [en línea]. Recuperado de: http://peremarques.pangea.org/calida2.htm

MATUSIAK, Krystyna K. (2013), Image and multimedia resources in an academic environment: A qualitative study of students' experiences and literacy practices. University of Denver. Journal of the American Society for Information Science and Technology 64 (8) 1577-1589. doi:10.1002/asi.22870. [en línea]. Recuperado de: https://digitalcommons.du.edu/cgi/viewcontent.cgi?article=1004\&context=lis_facpu $\mathrm{b}$

MINISTERIO DE LA PRESIDENCIA Y PARA LA ADMINISTRACIONES TERRITORIALES. Ley Orgánica de Educación (LOE). (BOE Núm. 106, 4-052006). Madrid - España. [en línea]. Recuperado de: https://www.boe.es/buscar/pdf/2006/BOE-A-2006-7899-consolidado.pdf

OLIVO ESTRADA, José Ramón y MONTAÑO TORRES, Carmelina (2014). Propuesta para desarrollar habilidades en investigación en la Universidad Autónoma de Nayarit, desde la investigación - acción. Revista Electrónica sobre Tecnología, Educación y Sociedad. Vol. 1. Núm 2. ISSN: 2448 - 6493. [en línea]. Recuperado de: http://www.ctes.org.mx/index.php/ctes/article/view/392/602

PODDER, Ranjiit. (2017). Teacher development for Quality Education. Associate Professor at Teachers' Training College, Dhaka. Published by Maynal Hossain Chowdhury on behalf of East West Media Group Limited. Magazine Daily Sun. [en línea]. Recuperado de: http://www.dailysun.com/printversion/details/265138/Teacher-Development-for-Quality-Education

RAMOS SÁNCHEZ, José Luis (2008). Reformas, investigación, innovación y calidad educativa. Revista Iberoamericana de Educación ISSN: 1681-5653. Editado por: Organización de Estados Iberoamericanos para la Educación, la Ciencia y la Cultura 
(OEI) Universidad de Extremadura, España. [en línea]. Recuperado de: file:///C:/Users/Docente01/Downloads/innovacion,investigacion\%20calidad.pdf

RED DE MAESTROS Y MAESTRAS POR LA REVOLUCIÓN EDUCATIVA (2016). Propuesta de la Comunidad Educativa como insumo para el nuevo Plan Decenal de Educación 2016-2025. febrero 2016. [en línea]. Recuperado de: https://educacion.gob.ec/wp-content/uploads/downloads/2016/03/PLAN-

DECENAL-PROPUESTA.pdf

SCOTT, Cynthia Luna. (2015). El futuro del aprendizaje 2 ¿Qué tipo de aprendizaje se necesita en el siglo XXI? Investigación y Prospectiva en Educación UNESCO, París. [Documentos de Trabajo ERF, No. 14]. [en línea]. Recuperado de: http://unesdoc.unesco.org/images/0024/002429/242996s.pdf

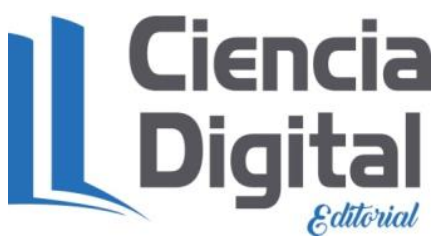


Para citar el artículo indexado.

Paredes M., Cruz Lascano M. \& Velasteguí E. (2018). Investigación e innovación como valor agregado para una educación superior de calidad. Revista electrónica Ciencia Digital 2(2), 216-231. Recuperado desde:

http://cienciadigital.org/revistacienciadigital/index.php/CienciaDigital/article/view/84

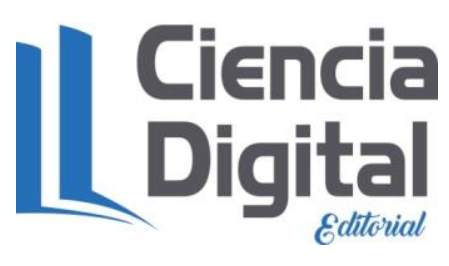

El artículo que se publica es de exclusiva responsabilidad de los autores y no necesariamente reflejan el pensamiento de la Revista Ciencia Digital.

El articulo queda en propiedad de la revista y, por tanto, su publicación parcial y/o total en otro medio tiene que ser autorizado por el director de la Revista Ciencia Digital.
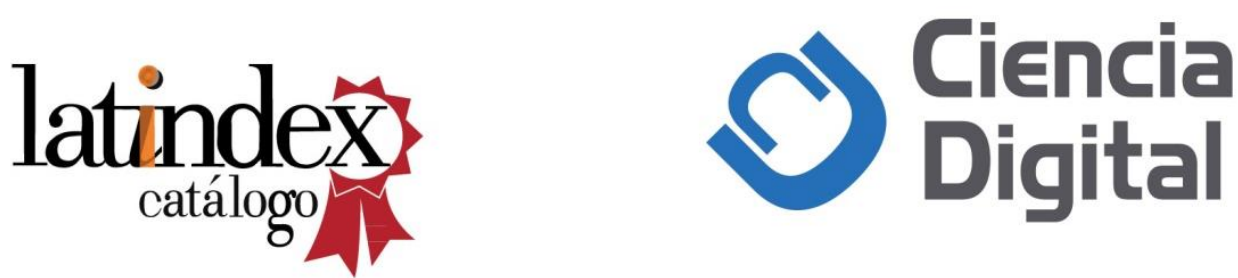